\title{
LA EVALUACIÓN ÉTICA EN LAS CIENCIAS HUMANAS Y/O SOCIALES. LA INVESTIGACIÓN CIENTÍFICA: ENTRE LA LIBERTAD Y LA RESPONSABILIDAD
}

\author{
M. Lucrecia Rovaletti*
}

Resumen: Este artículo analiza la situación de la evaluación ética de proyectos de investigación en las ciencias sociales y/o humanas. En las evaluaciones de proyectos pertenecientes a estas áreas, el análisis de los requisitos éticos no se considera necesario o no se pone una atención tan estricta como en materias biomédicas. Sin embargo, las diferencias entre proyectos clínico-farmacológicos y de ciencias humanas y/o sociales exigen una evaluación ética específica para estos últimos, de modo de resguardar los principios de beneficencia, no maleficencia, autonomía y justicia.

Palabras clave: ética de la investigación, ciencias sociales y/o humanas, libertad, responsabilidad

\section{ETHICAL REVIEW IN HUMANITIES AND SOCIAL SCIENCES. SCIENTIFIC RESEARCH: BETWEEN FREEDOM AND RESPONSIBILITY}

Abstract: This paper analyzes the situation of the ethical review of research projects in social sciences and humanities. In the review of research projects belonging to these fields, it has been found that the analysis of ethical requirements are not considered necessary or there is no close attention devoted to it as in biomedical projects. Nevertheless, the differences between clinical-pharmacological projects and the humanities and social sciences demand a specific ethical review for the latter, so that the principles of beneficence, non maleficence, autonomy and justice are safeguarded.

Key words: ethics of research, social sciences and/or humanities, freedom, responsibility

\section{A AVALIAÇÃO ÉTICA NAS CIÊNCIAS HUMANAS E/OU SOCIAIS. A PESQUISA CIENTÍFICA ENTRE A LIBERDADE E A RESPONSABIIDADE}

Resumo: Este artigo analisa a situação da avaliação ética de projetos de pesquisa nas ciências sociais e/ou humanas. Nas avaliações de projetos destas áreas, a análise dos requisitos éticos não se considera necessária ou não se dá uma atenção específica como nas questões biomédicas. Contudo, as diferenças entre projetos clínico-farmacológicos e de ciências humanas e/ou sociais, exigem uma avaliação ética específica para estas últimos, procurando salvaguardar os princípios de beneficencia, não maleficência, autonomia e justiça.

Palavras chave: ética da pesquisa, ciencias sociais e/ou humanas, liberdade, responsabilidade

* Investigadora Principal del CONICET, Profesora Regular de la Universidad de Buenos Aires. Argentina Correspondencia:mlrova@psi.uba.ar 


\section{De la comunidad científica a la comunidad moral}

Al comienzo de su Metafísica, Aristóteles afirma que "todos los hombres están deseosos de saber". Tiempo después, Freud traducirá esta orientación en una pulsión de apropiación de lo otro como objeto, que se sublima voluntariamente en un impulso de ver y saber. En la época actual son muchos los que arrojan una sospecha sobre esta libido cognoscendi, que ha devenido finalmente una libido dominandi. En efecto, allí se combinan una valorización legítima del conocer con el presentimiento de un avance científico potencialmente peligroso, tanto para el sujeto cognoscente como para el objeto conocido.

¿Cómo es posible entonces mantener un equilibrio entre la libertad y/o la obligación del conocimiento y el bien público? $(1, p .65)$. El problema ético y filosófico se ubica exactamente en este punto. "Cuando se comienza a tratar al hombre según los criterios de la cientificidad 'objetiva' (...) se transforma efectivamente al ser humano en objeto y, como consecuencia, se lo suprime en tanto ser humano"(2, p.289).

¿La utilización social del conocimiento basta para su aceptabilidad, como piensan Sábato, Varsawsky y Herrera? Aunque el mito de la "neutralidad" del hecho científico siga impregnando la práctica social de la ciencia como profesión, no todo "lo factible en ciencia" es siempre socialmente legítimo(3, p.146).

Hay algo más, se trata de responder si existe el derecho de hacer progresar el conocimiento o se postula una libertad condicionada; se debate si la búsqueda cognitiva justifica moralmente que se apliquen al ser humano procedimientos de investigación o experimentación que puedan ser agresivos, arriesgados o indiscretos. Anne Langlois considera que las comisiones de ética ejercen un rol tranquilizador que funciona como barrera de contención ante esta "angustia cultural". Se trata de compatibilizar el conocimiento con los otros valores con los cuales relacionamos nuestras elecciones éticas: el bienestar, la libertad y la justicia.

Por lo expuesto, la ciencia se ha convertido en un asunto que atañe a la sociedad toda, porque ésta adhiere a la investigación en la medida en que es compatible con los derechos del hombre. El respeto a la "dignidad de la ciencia" es parte, sin duda, del respeto a la dignidad humana, pero la coherencia entre estos dos aspectos no siempre es evidente, $\mathrm{y}$ la intuición más común dicta que el deber de proteger a las personas humanas fije los límites del conocer.

La investigación debe estar gobernada por normas y valores éticos, aun cuando no exista acuerdo sobre qué normas éticas son aplicables. Algunas normas expresan valores éticos generales que son, sin duda, fundamentales. Otras veces las perspectivas éticas no son tan claras ni han sido tan ponderadas; incluso pueden contradecirse entre sí. Esta falta de claridad y acuerdo surge porque la investigación nos brinda oportunidades y conocimientos nuevos respecto de los cuales no podemos tomar una posición ética de inmediato.

Sin embargo, mientras la comunidad científica se defina sólo por criterios de "excelencia" no podrá lograse una comunidad moral, y los científicos, a falta de competencia ética, siempre estarán expuestos a ser recusados a título diverso. Además, las instituciones académicas están obligadas a evaluar no sólo metodológica, sino también éticamente toda investigación que así lo requiera, estableciendo normativas claras y de fácil acceso.

Al referirse al proceso de investigación científica, Fernando Lolas (3, p.143) distingue tres aspectos: el técnico o instrumental, que incluye 
las características y justificación de los métodos empleados, la exactitud de las mediciones y la aceptación de los enunciados por la comunidad de pares; el cientifico o hermenéutico, que alude a la capacidad "fertilizadora" que pueda tener una propuesta de investigación para su propia disciplina o para otras, es decir, la "utilidad" de los conceptos que produce (invención) o las innovaciones de forma y fondo que introduce en la praxis científica, y, en tercer término, el aspecto social o emancipatorio, que indica el impacto de una propuesta (o sus productos) en la sociedad general, esto es, más allá de los expertos o pares y de otros científicos. Por tanto, un proyecto de investigación, en tanto proceso social, puede ser examinado como procedimiento técnico y como producto intelectual empleando estas tres dimensiones.

Ahora bien, el análisis de la institución social de la ciencia suele realizarse por dos grupos de personas: por quienes profesionalmente se dedican a ella, la llamada "scientific communitiy", que puede equipararse a la "opinión pública" de la comunidad cognoscitiva y social investigadora; luego, por el juicio externo de los usuarios de productos científicos, planificadores de la actividad científica, políticos, industriales(4). Para ambos grupos rigen distintas racionalidades y, por tanto, una diversa ponderación de las dimensiones técnica, científica y social. Mientras la scientific community probablemente valore más las dos primeras, la comunidad general se orientará a la tercera.

Las pautas éticas en la investigación tienen como objetivo ayudar a todo investigador y a la comunidad de investigadores: a reflexionar sobre sus perspectivas y actitudes éticas; a tener en cuenta que existen reglas que, entre sí, pueden entrar en conflicto, y a capacitarse para decidir de manera fundamentada. En consecuencia, estas pautas no se limitan a las normas que atañen al investigador y a directivos de las instituciones de investigación, sino que también contienen normas para otras organizaciones que puedan tener influencia sobre sus consecuencias.

Estas pautas no tienen las mismas funciones que las leyes. Más bien se ocupan de la cautela y protección, y sustentan o complementan las actuales normas jurídicas, aunque a veces se superponen con ellas. En una democracia donde impera el Estado de Derecho, los científicos, al igual que otros miembros de la sociedad, están sujetos a la ley.

Las obligaciones éticas de la investigación se refieren, en parte, a las normas que rigen el proceso de investigación $\mathrm{y}$, en parte, a la responsabilidad sobre sus consecuencias. De allí la necesidad de contar con una evaluación ética.

\section{Las investigaciones en ciencias sociales y/o humanas y la necesidad de una evaluación ética}

¿Cuál es la situación en las ciencias sociales y/o humanas respecto de la evaluación ética de proyectos de investigación?

- En la literatura actual, cuando se señalan problemas éticos, una gran parte se relaciona con el ejercicio profesional y sólo una pequeña con investigación. En Internet, sin embargo, aparece un interesante material proveniente de los recientes comités de investigación en ciencias sociales y/o humanas.

- En los seminarios, jornadas y cursos sobre pautas éticas de investigación, los comités de evaluación de proyectos en ciencias sociales y humanas no constituyen todavía un tema.

- En los comités de ética de investigación clínica y farmacológica, que residen en su mayoría en centros hospitalarios, los aspec- 
tos psicoéticos son soslayados o se diluyen en problemas generales; lo mismo ocurre en las otras áreas de las ciencias sociales. En relación con proyectos de investigación pertenecientes a estas áreas la valoración ética no se considera un requisito.

En efecto, partiendo de una concepción dualista, se piensa que el riesgo para los participantes, a diferencia de la investigación médica, es casi nulo, dado que no implica daño físico. Más aún, se estima que, en este ámbito, resulta difícil definir y medir el daño potencial, es decir, evaluar sus efectos a largo plazo.

En caso de demandar una evaluación ética, se exige a estas investigaciones los mismos tipos de protocolos que las ciencias biomédicas, sin reparar ni respetar las diferentes metodologías ni reconocer que los objetos formales que se investigarán presentan características diferentes. Ya no se trata de meros "sujetos" de investigación sino de "participantes" en ella: más que una investigación "sobre" el hombre devendría en una investigación "con" el hombre. En estas áreas del saber los humanos dejan de ser "objetos" pasivos de una dirección cognitiva reductora para convertirse en "sujetos" activos del esfuerzo de conocimiento que les concierne.

¿Podrá la investigación científica actual considerar nuevas alianzas entre las tareas científicas y las demandas humanas? "Urge plantear una 'comunidad científica ampliada' que permita a los nuevos actores sociales una apropiación colectiva de los saberes $(5, p .109)$.

\section{La especificidad de los proyectos de ciencias humanas y/o sociales}

Se trata ahora de analizar las diferencias significativas entre los proyectos clínico-farmacológicos y los de ciencias humanas y/o sociales, para mostrar la especificidad de estos últimos, amenazados en su diversidad y en sus metodología por la hegemonía biomédica y experimental.

Los proyectos de investigación en el campo clínico y farmacológico son, en general, multicéntricos, están subsidiados por la industria pero son evaluados científica y éticamente por las instituciones donde se realiza la investigación (en general hospitales públicos). Los proyectos que se realizan en el campo de las ciencias sociales y/o humanas, en cambio, están subsidiados por instituciones académicas y, a veces, ONG que los evalúan científica, pero no éticamente. Por su parte, aquellos variados ámbitos donde se realiza la investigación (poblaciones e instituciones pequeñas, colegios, cárceles, entre otros) suponen y confían en el aval global de las instituciones que aprobaron el proyecto, sin distinguir los aspectos científicos de los éticos.

Si a esta situación se añaden algunos proyectos de investigación de público conocimiento en el área académica, como el caso Milgram, Tronick, Berkun, Bramel, Golbert y otros $(6,7)$, que fueron aprobados por instituciones académicas, a pesar de que no cumplían con los requisitos éticos, se hace patente la necesidad de una instancia de evaluación en esta área de las ciencias humanas. Como señala FrançaTarrago(8), en las nuevas pautas de los organismos oficiales "se debe subsanar la excesiva insistencia en los aspectos de la salud física y la minusvalía" que se da en "la investigación relacionada con la salud mental o conductual de los seres humanos".

El aumento de las investigaciones en estas disciplinas hace necesario realizar una evaluación ética de sus protocolos, para que se respeten los principios de beneficencia (qué resultados se lograrán), no-maleficencia (examen costo-beneficio, aseguramiento ante posibles perjuicios, protección de los sujetos vulnerables), autonomía (información acerca 
del objetivo de la investigación, consentimiento esclarecido o validado, coerción en la participación respeto de subordinados, respeto a la intimidad y singularidad étnica y/o grupal, confidencialidad y resguardo de datos, posibilidad de abandonar la investigación) y justicia (respeto a la diversidad local, equidad en la selección de la muestra).

Las nuevas exigencias de evaluación ética requieren de una guía que permita identificar estos conflictos, con el fin de ser utilizada a la hora de formular estos proyectos. De allí la necesidad de contar con un tipo de instrumento que pueda también dar cuenta específicamente de aquellas investigaciones con temáticas propias de América Latina.

Ahora bien, dado el tiempo que puede insumir la constitución de comités de ética de la investigación en el campo "Psi" -por el debate que implica su aprobación, caracterización e implementación-, una guía que permita identificar conflictos éticos en la formulación y ejecución de proyectos por parte de los investigadores -y para la evaluación de los mismos por parte de las instituciones académicas- puede cubrir en mejores condiciones el período que lleve la constitución de estos comités.

\section{a) Garantizar la libertad para investigar}

Las universidades e instituciones académicas son especialmente responsables de garantizar la libertad de investigar. Esto implica que las investigaciones deben ser libres, críticas e innovadoras, y deben otorgarse todas las garantías para que no se impida ni se interrumpa ningún proyecto por el solo hecho de que su tema resulte polémico. El investigador debe verse libre tanto de los controles que le impidan plantear problemas que supuestamente se oponen a ciertos intereses (económicos, políticos y sociales) como de la supresión de los resultados y las conclusiones.
No se debe permitir que los intereses de los patrocinadores -tanto del sector público como privado-tengan mayor peso que la exigencia de un informe veraz, ni que se retengan los resultados de una investigación por ser contrarios a esos intereses, o que se extraigan determinadas conclusiones que puedan brindar una imagen distorsionada de la misma. En ciertas circunstancias se debe recurrir al derecho de suspender una investigación por incumplimiento del contrato.

Es imprescindible que se proteja la investigación de aquellas presiones que amenazan con socavar los estándares del método científico, como ocurre cuando se otorga confiabilidad científica a metodologías espurias.

Independientemente de sus decisiones y elecciones personales, el investigador, como miembro de una comunidad científica, tiene que defender ante los actores sociales la idea de que la pesquisa desinteresada es parte del enriquecimiento cultural de la sociedad y que el saber científico contribuye al desarrollo de nuestra civilización. La libertad de investigar demanda de cada investigador y de la comunidad científica la voluntad y la capacidad para cumplir con las exigencias éticas.

\section{b) La responsabilidad del científico como agente ético}

La libertad del investigador se sostiene y se mantiene con el cumplimiento de sus responsabilidades. El concepto de responsabilidad se asocia al de imputabilidad, es decir, a la posibilidad de evaluar éticamente ciertas conductas. Ahora bien, un sujeto sólo es imputable por comisión o por omisión, si por su competencia tenía el deber de conocer las consecuencias de los medios utilizados para lograr unos fines éticamente justificables. "No basta decir que un determinado sujeto debe responder con determinada tarea, sino que debe hacerlo, actuando 
libre, razonada y prudentemente. Es decir, la forma de llevar a cabo la tarea imputable por el sujeto imputable debe ser ella misma imputable de responsabilidad"(8).

En este sentido, los científicos, como agentes éticos, son responsables de informar a la sociedad de las consecuencias de determinadas investigaciones o de utilizar ciertos medios o técnicas para lograr tales objetivos. Frente a los participantes deben definir los límites, expectativas y requisitos que son propios de su papel de investigador.

Si bien los investigadores están potencialmente interesados en todos los aspectos del comportamiento humano, existen razones éticas que impiden trascender algunas áreas de la experiencia y conducta, ya sea por metodologías experimentales, observacionales u otra forma de investigación psicológica. Por eso, en toda circunstancia deben tenerse en cuenta las implicaciones éticas y las consecuencias psicológicas y sociales que puedan ejercerse para los participantes ${ }^{1}$.

El investigador debe asegurar la libertad y autodeterminación del participante, dado que éste se encuentra, de algún modo, sujeto a la voluntad del proyecto científico y a la posibilidad de manipulación que esa sujeción implica. Esta situación puede llevarlo a sentirse degradado, al estar en juego su autoestima u otros valores de importancia para él. Los investigadores no deben olvidar que están en deuda con aquellos que acuerdan participar en sus estudios, porque están dispuestos a dar su tiempo (incluso cuando hay remuneración) y hasta correr algún riesgo, por lo que merecen ser tratados con los más altos estándares de consideración y respeto.

1 En algunos países, alegando que se han infringido los derechos y dignidad de los participantes, han aumentado recientemente las acciones legales contra los profesionales. Prueba de ello es la existencia de seguros para la praxis profesional.
Cuando se trata de analizar comportamientos y valores de minorías -como grupos religiosos, minorías étnicas, grupos de jóvenes o subculturas políticas y marginados- hay que ponderar que éstos pueden sentir que la investigación los hace más vulnerables a la desaprobación social. Ello obliga a un cuidadoso respeto por sus creencias, opiniones y modos de vida, aun cuando éstos difieran de los aceptados por la mayoría. Además, se debe ser prudente con las descripciones a fin de evitar prejuicios en su autoimagen y en los derechos que los asisten como minorías.

También las personas fallecidas nos exigen, a su modo, respeto por su reputación, para lo cual es preciso elegir con cautela los términos que se emplean para referirse a ellas.

\section{c) Frente a la sociedad}

Al difundir los resultados de la investigación, el investigador debe expresarse con la mayor claridad posible, a fin de evitar malentendidos y/o equivocadas interpretaciones; también debe ser especialmente cauteloso al publicar resultados que no han sido suficientemente discutidos y evaluados en círculos de investigación.

\section{d) Frente a la comunidad de investigadores}

El material de investigación debe estar disponible para que otros investigadores puedan verificarlo, o volver a analizarlo y utilizarlo. El investigador debe respetar el trabajo de otros, citando las fuentes de sus ideas, como publicaciones o materiales de investigación. Algo similar deben tener en cuenta los directores respecto de sus dirigidos o becarios, reconociendo las aportaciones personales de los mismos.

La responsabilidad alcanza a los propios investigadores cuando, como evaluadores, tienen que dictaminar sobre cuestiones científicas. 
Sería el caso de postulantes para cargos científicos, tesis doctorales y de posgrado, aplicaciones de proyectos, presentaciones de trabajos a revistas científicas, evaluación de protocolos hospitalarios y otras cuestiones similares. Ellos están obligados a aplicar estándares académicos razonables, promoviendo un debate objetivo sobre aplicaciones y limitaciones que tienen los diversos métodos.

Pero ello debe estar acompañado de una actitud abierta frente a las distintas metodologías, a las diversas áreas de investigación y a las diferentes perspectivas y orientaciones. Además, los miembros o jurados de esas comisiones dictaminadoras deben estar dispuestos a oír los argumentos provenientes por otras escuelas de pensamiento; dicha apertura demostrará si son competentes o no para esa tarea.

La diversidad de escuelas y orientaciones no debe ser obstáculo para que se trabaje según normas fundadas y reconocidas. También es necesario que, durante la realización de las investigaciones, se hagan cumplir esos mismos estándares, controlando su calidad. Cuando esto no acaece, no deben silenciarse las críticas, esgrimiendo razones de lealtad o de obediencia. Sólo el juicio constructivo y el intercambio de información permiten alcanzar, en cada ámbito, verdaderos estándares científicos.
Los estándares que deben cumplirse y la validez de los razonamientos cobran particular importancia cuando la investigación puede acarrear consecuencias para la reputación o integridad de individuos o grupos, o pudiera influir en decisiones políticas. En estos casos, es de gran importancia que el investigador presente posibles interpretaciones alternativas de sus descubrimientos.

\section{Las organizaciones de las ciencias psicológicas y los organismos internacionales}

En el campo preciso de las ciencias de la conducta, França-Tarragó ${ }^{2}$ ha planteado que en las pautas CIOMS no se han explicitado suficientemente las dificultades psicológicas que puedan surgir en la investigación con seres humanos. En efecto, si bien no excluyen la perspectiva de la salud mental como objeto de investigación, hay una excesiva "medicalización" del tema dejando de lado los aportes fundamentales de la psicología. No hace falta recordar que la OMS incluye en la definición de la salud los aspectos psicológicos. Por ello, estas normativas deberían insistir que en toda investigación en salud mental o conductual el diseño sea sometido a la evaluación de los comités de ética respectivos.

2 "Quizá haya llegado el momento en que a la CIOMS se agreguen las organizaciones de las ciencias psicológicas. O, por el contrario, que la Organización Mundial de la Salud anime a la conformación de una organización específica para los aspectos de la salud mental o psicológica de los seres humanos, que se ocupe de afrontar los dilemas propios de la investigación con este tipo de personas". França-Tarragó O. Algunas dificultades en la aplicabilidad de las Pautas Éticas en los países de América Latina. Presentado en el seminario "Pautas Éticas Internacionales para la Investigación Biomédica en Seres Humanos. Nuevos desarrollos". Santiago de Chile, 16 y 17 de octubre de 2003. 


\section{Referencias}

1. Jonas H. Técnica, medicina y ética. La práctica del principio de responsabilidad. Barcelona: Paidós; 1997.

2. Ladrière J. Expérimentation humaine et droits de l'homme. En : Experimentation biomédicale et droits de l'homme. Paris : PUF; 1988.

3. Lolas F. Bioética y antropología médica. Santiago de Chile: Editorial Mediterráneo; 2000: 143-150.

4. Benson PR. The social control of biomedical research. Social Science and Medicine 1989; 29: 1-12.

5. Rovaletti ML. La investigación biomédica actual: un cuestionamiento a la sociedad en su conjunto. Acta Bioethica 2003; 9(1): 105-111.

6. Schuler H. Ethical problems of psychological research. New Cork: Academic Press; 1982: 88.

7. Leibovich de Duarte AS. La dimensión ética en la investigación psicológica. Investigaciones en Psicología 2000; 5(1): 41-61.

8. França-Tarragó O. Ética para Psicólogos. Bilbao: Desclée De Brouwer; 1996.

Recibido el 16 de noviembre de 2005.

Aceptado el 04 de enero de 2006. 